Research Article

\title{
The Mellin Transform of Logarithmic and Rational Quotient Function in terms of the Lerch Function
}

\author{
Robert Reynolds $(\mathbb{D}$ and Allan Stauffer \\ Department of Mathematics and Statistics, York University, Toronto, Canada M3J1P3 \\ Correspondence should be addressed to Robert Reynolds; milver@my.yorku.ca
}

Received 2 May 2021; Revised 7 July 2021; Accepted 22 July 2021; Published 4 August 2021

Academic Editor: Barbara Martinucci

Copyright (c) 2021 Robert Reynolds and Allan Stauffer. This is an open access article distributed under the Creative Commons Attribution License, which permits unrestricted use, distribution, and reproduction in any medium, provided the original work is properly cited.

Upon reading the famous book on integral transforms volume II by Erdeyli et al., we encounter a formula which we use to derive a Mellin transform given by $\int_{0}^{\infty}\left(\left(x^{m-1} \log ^{k}(a x)\right) /\left(\left(\beta^{2}+x^{2}\right)(c+x)\right)\right) \mathrm{d} x$, where the parameters $a, k$, $\beta$, and $\gamma$ are general complex numbers. This Mellin transform will be derived in terms of the Lerch function and is not listed in current literature to the best of our knowledge. We will use this transform to create a table of definite integrals which can be used to extend similar tables in current books featuring such formulae.

\section{Introduction}

The well-known book on Mellin transforms by Brychkov et al. [1] contains a vast number of integral formulae. In this work, we focus on adding to section (2.5.1) featuring "logarithmic and algebraic functions." In particular, this work will derive a closed form solution for the integral given by

$$
\int_{0}^{\infty} \frac{x^{m-1} \log ^{k}(a x)}{\left(\beta^{2}+x^{2}\right)(\gamma+x)} \mathrm{d} x,
$$

in terms of the Lerch function. This is achieved by applying our contour integration method to an integral transform from Erdeyli's book [2] to yield a definite integral in terms of the Lerch function. We have also produced other formulae in terms of the Lerch function using our method in [3]. We use this new integral transform in terms of the Lerch function to derive new formulae for Brychkov's book [1] thereby extending the current tables in section (2.5.1). We will also derive new Malmsten-type $\log (\log (x))$ integrals in terms of special functions and provide new formula also extending the work done by Malmsten [4]. The derivations of the formulae in this work follow the method used by us in [5]. This method involves using the generalized Cauchy's integral formula given by

$$
\frac{y^{k}}{k !}=\frac{1}{2 \pi i} \int \frac{e^{w y}}{C w^{k+1}} \mathrm{~d} y,
$$

and multiplying both sides of (2) by a function, followed by taking a definite integral on both sides which yields a definite integral in terms of a contour integral. Then, we multiply both sides of equation (2) by another function and take the infinite sum of both sides such that the contour integral of both equations are the same. Here, $C$ is, in general, an open contour in the complex plane where the bilinear concomitant has the same value at the end points of the contour.

\section{Definite Integral of the Contour Integral}

We use the method in [5]. The variable of integration in the contour integral is $\alpha=m+w$. The cut and contour are in the first quadrant of the complex $\alpha$-plane with $0<\operatorname{Re}(\alpha)<u$. The cut approaches the origin from the interior of the first quadrant and the contour goes round the origin with zero radius and is on opposite sides of the cut. Using a generalization of Cauchy's integral formula, we first replace $y$ by $\log (a x)$ followed by multiplying both sides by

$$
\frac{x^{m-1}}{\left(\beta^{2}+x^{2}\right)(\gamma+x)},
$$


and then taking the definite integral with respect to $x \in[0, \infty)$ to obtain

$$
\begin{aligned}
\frac{1}{k !} \int_{0}^{\infty} \frac{x^{m} \log ^{k}(a x)}{\left(\beta^{2}+x^{2}\right)(\gamma+x)} \mathrm{d} x= & \frac{1}{2 \pi i} \int_{0}^{\infty} \int_{C\left(\beta^{2}+x^{2}\right)(\gamma+x)} \frac{a^{w} w^{-k-1} x^{m+w}}{2} \mathrm{~d} x \\
= & \frac{1}{2 \pi i} \int_{C} \int_{0}^{\infty} \frac{a^{w} w^{-k-1} x^{m+w}}{\left(\beta^{2}+x^{2}\right)(\gamma+x)} \mathrm{d} x \mathrm{~d} w \\
= & \frac{1}{2 \pi i} \int_{C} \frac{\pi a^{w} w^{-k-1}}{2\left(\beta^{2}+\gamma^{2}\right)}\left(\gamma \beta^{m+w-1} \sec \left(\frac{1}{2} \pi(m+w)\right)\right. \\
& +\beta^{m+w} \csc \left(\frac{1}{2} \pi(m+w)\right) \\
& \left.-2 \gamma^{m+w} \csc (\pi(m+w))\right) \mathrm{d} w
\end{aligned}
$$

from equation (3.263) in [6], where $-1<\operatorname{Re}(m+w)<2$ and $\operatorname{Re}(\beta)>0,|\arg (\gamma)|<\pi$. We are able to switch the order of integration over $w$ and $x$ using Fubini's theorem since the integrand is of bounded measure over the space $\mathbb{C} \times[0, \infty)$.

\section{The Lerch Function}

The Hurwitz-Lerch function [7] has a series representation given by

$$
\Phi(z, s, v)=\sum_{n=0}^{\infty}(v+n)^{-s} z^{n}
$$

where $v \in \mathbb{C} \backslash \mathbb{Z}_{0}^{-} ; s \in \mathbb{C}$ when $|z|<1 ; \operatorname{Re}(s)>1$ when $|z|=1$ and is continued analytically by its integral representation given by

$$
\begin{aligned}
\Phi(z, s, v) & =\frac{1}{\Gamma(s)} \int_{0}^{\infty} \frac{t^{s-1} e^{-v t}}{1-z e^{-t}} \mathrm{~d} t \\
& =\frac{1}{\Gamma(s)} \int_{0}^{\infty} \frac{t^{s-1} e^{-(v-1) t}}{e^{t}-z} \mathrm{~d} t
\end{aligned}
$$

where $\operatorname{Re}(v)>0$ and either $|z| \leqq 1, z \neq 1, \operatorname{Re}(s)>0 ; z=1$, $\operatorname{Re}(s)>1$.

\section{Infinite Sum of the Contour Integrals}

4.1. Derivation of the First Contour Integral. In this section, we will derive the Lerch function representation for the first contour integral given by

$$
\frac{1}{2 \pi i} \int_{C} \frac{\pi a^{w} w^{-k-1} \beta^{m+w} \csc ((1 / 2) \pi(m+w))}{2\left(\beta^{2}+\gamma^{2}\right)} \mathrm{d} w .
$$

Using equation (2) and replacing $y$ by $\log (a)+\log (\beta)+$ $(1 / 2) i \pi(2 y+1)$ and then multiplying both sides by

$$
-\frac{i \pi \beta^{m} e^{(1 / 2) i \pi m(2 y+1)}}{\beta^{2}+\gamma^{2}},
$$

we obtain

$$
\begin{aligned}
& -\frac{i(i / 2)^{k} \pi^{k+1} \beta^{m} e^{i \pi m y+(i \pi m / 2)}}{k !\left(\beta^{2}+\gamma^{2}\right)}\left(-\frac{2 i \log (a)}{\pi}-\frac{2 i \log (\beta)}{\pi}+2 y+1\right)^{k} \\
& =-\frac{1}{2 \pi i} \int_{C} \frac{i \pi w^{-k-1} \beta^{m}}{\beta^{2}+\gamma^{2}} \exp \left(w\left(\log (a)+\log (\beta)+\frac{1}{2} i \pi(2 y+1)\right)\right. \\
& \left.+\frac{1}{2} i \pi m(2 y+1)\right) \mathrm{d} w .
\end{aligned}
$$

We then take the infinite sum over $y \in[0, \infty)$ simplifying in terms of the Lerch function to obtain 


$$
\begin{aligned}
& -\frac{(i \pi)^{k+1} e^{(i \pi m / 2)} \beta^{m} \Phi\left(e^{i m \pi},-k,\left(\left(-2 i \log (a)-t 2 n i q h_{l} \operatorname{og} x(\beta)\right)+\pi / 2 \pi\right)\right)}{k !\left(\beta^{2}+\gamma^{2}\right)} \\
= & -\frac{1}{2 \pi i} \sum_{y=0}^{\infty} \int_{C} \frac{i \pi w^{-k-1} \beta^{m}}{\beta^{2}+\gamma^{2}} \exp \left(w\left(\log (a)+\log (\beta)+\frac{1}{2} i \pi(2 y+1)\right)+\frac{1}{2} i \pi m(2 y+1)\right) \mathrm{d} w \\
= & -\frac{1}{2 \pi i} \int_{C} \sum_{y=0}^{\infty} \frac{i \pi w^{-k-1} \beta^{m}}{\beta^{2}+\gamma^{2}} \exp \left(w\left(\log (a)+\log (\beta)+\frac{1}{2} i \pi(2 y+1)\right)+\frac{1}{2} i \pi m(2 y+1)\right) \mathrm{d} w \\
= & \frac{1}{2 \pi i} \int_{C} \frac{\pi a^{w} w^{-k-1} \beta^{m+w} \csc ((1 / 2) \pi(m+w))}{2\left(\beta^{2}+\gamma^{2}\right)} \mathrm{d} w,
\end{aligned}
$$

from (1.232.3) in [6], where $\operatorname{Im}(m+w)>0$ in order for the sum to converge, and $\operatorname{csch}(i x)=-i \csc (x)$ from (4.5.10) in [8].

4.2. Derivation of the Second Contour Integral. In this section, we will derive the Lerch function representation for the second contour integral given by

$$
-\frac{1}{2 \pi i} \int_{C} \frac{\pi a^{w} w^{-k-1} \gamma^{m+w} \csc (\pi(m+w))}{\beta^{2}+\gamma^{2}} \mathrm{~d} w .
$$

Using equation (2) and replacing $y$ by $\log (a)+\log (\gamma)+$ $i \pi(2 y+1)$ and then multiplying both sides by

$$
\frac{2 i \pi \gamma^{m} e^{i \pi m(2 y+1)}}{\beta^{2}+\gamma^{2}}
$$

we obtain

$$
\begin{aligned}
& \frac{2 i i^{k} \pi^{k+1} \gamma^{m} e^{2 i \pi m y+i \pi m}}{k !\left(\beta^{2}+\gamma^{2}\right)}\left(-\frac{i \log (a)}{\pi}-\frac{i \log (\gamma)}{\pi}+2 y+1\right)^{k} \\
& =-\frac{1}{2 \pi i} \int_{C} \frac{2 i \pi w^{-k-1} \gamma^{m}}{\beta^{2}+\gamma^{2}} \exp (w(\log (a)+\log (\gamma)+i \pi(2 y+1))+i \pi m(2 y+1)) \mathrm{d} w .
\end{aligned}
$$

We then take the infinite sum over $y \in[0, \infty)$ simplifying in terms of the Lerch function to obtain

$$
\begin{aligned}
& \frac{(2 i \pi)^{k+1} e^{i \pi m} \gamma^{m} \Phi\left(e^{2 i m \pi},-k,((-i \log (a)-\operatorname{tin} q h \log (\gamma))+\pi / 2 \pi)\right)}{k !\left(\beta^{2}+\gamma^{2}\right)} \\
& =-\frac{1}{2 \pi i} \sum_{y=0}^{\infty} \int_{C} \frac{2 i \pi w^{-k-1} \gamma^{m}}{\beta^{2}+\gamma^{2}} \exp (w(\log (a)+\log (\gamma)+i \pi(2 y+1))+i \pi m(2 y+1)) \mathrm{d} w \\
& =-\frac{1}{2 \pi i} \int_{C} \sum_{y=0}^{\infty} \frac{2 i \pi w^{-k-1} \gamma^{m}}{\beta^{2}+\gamma^{2}} \exp (w(\log (a)+\log (\gamma)+i \pi(2 y+1))+i \pi m(2 y+1)) \mathrm{d} w \\
& =-\frac{1}{2 \pi i} \int_{C} \frac{\pi a^{w} w^{-k-1} \gamma^{m+w} \csc (\pi(m+w))}{\beta^{2}+\gamma^{2}} \mathrm{~d} w,
\end{aligned}
$$


from $(1.232 .3)$ in [6], where $\operatorname{Im}(m+w)>0$ in order for the sum to converge, and $\operatorname{csch}(i x)=-i \csc (x)$ from (4.5.10) in [8].

4.3. Derivation of the Third Contour Integral. In this section, we will derive the Lerch function representation for the third contour integral given by

$$
-\frac{1}{2 \pi i} \int_{C} \frac{\pi \gamma a^{w} w^{-k-1} \beta^{m+w-1} \sec ((1 / 2) \pi(m+w))}{2\left(\beta^{2}+\gamma^{2}\right)} \mathrm{d} w .
$$

Using equation (2) and replacing $y$ by $\log (a)+\log (\beta)+$ $(1 / 2) i \pi(2 y+1)$ and then multiplying both sides by

$$
\frac{\pi \gamma(-1)^{y} \beta^{m-1} e^{(1 / 2) i \pi m(2 y+1)}}{\beta^{2}+\gamma^{2}},
$$

we obtain

$$
\begin{array}{r}
\frac{\gamma(i / 2)^{k} \pi^{k+1}(-1)^{y} \beta^{m-1} e^{i \pi m y+(i \pi m / 2)}}{k !\left(\beta^{2}+\gamma^{2}\right)}\left(-\frac{2 i \log (a)}{\pi}-\frac{2 i \log (\beta)}{\pi}+2 y+1\right)^{k} \\
=\frac{1}{2 \pi i} \int_{C} \frac{\pi \gamma(-1)^{y} w^{-k-1} \beta^{m-1}}{\beta^{2}+\gamma^{2}} \exp \left(w\left(\log (a)+\log (\beta)+\frac{1}{2} i \pi(2 y+1)\right)+\frac{1}{2} i \pi m(2 y+1)\right) \mathrm{d} w .
\end{array}
$$

We then take the infinite sum over $y \in[0, \infty)$ simplifying in terms of the Lerch function to obtain

$$
\begin{aligned}
& \frac{\gamma \pi^{k+1} e^{(1 / 2) i \pi(k+m)} \beta^{m-1}}{k !\left(\beta^{2}+\gamma^{2}\right)} \Phi\left(-e^{i m \pi},-k,((-2 i \log (a)-2 i \log (\beta)+\pi) / 2 \pi)\right) \\
& =\frac{1}{2 \pi i} \sum_{y=0}^{\infty} \int_{C} \frac{\pi \gamma(-1)^{y} w^{-k-1} \beta^{m-1}}{\beta^{2}+\gamma^{2}} \exp \left(w(\log (a)+\log (\beta)+(1 / 2) i \pi(2 y+1))+\frac{1}{2} i \pi m(2 y+1)\right) \mathrm{d} w \\
& =\frac{1}{2 \pi i} \int_{C} \sum_{y=0}^{\infty} \frac{\pi \gamma(-1)^{y} w^{-k-1} \beta^{m-1}}{\beta^{2}+\gamma^{2}} \exp \left(w\left(\log (a)+\log (\beta)+\frac{1}{2} i \pi(2 y+1)\right)+\frac{1}{2} i \pi m(2 y+1)\right) \mathrm{d} w \\
& =\frac{1}{2 \pi i} \int_{C} \frac{\pi \gamma a^{w} w^{-k-1} \beta^{m+w-1} \sec ((1 / 2) \pi(m+w))}{2\left(\beta^{2}+\gamma^{2}\right)} \mathrm{d} w,
\end{aligned}
$$


from (1.232.2) in [6], where $\operatorname{Im}(m+w)>0$ in order for the sum to converge, and $\operatorname{sech}(i x)=i \sec (x)$ from (4.5.11) in [8].

\section{Definite Integral in terms of the Lerch Function}

Theorem 1. For $k, a, m \in \mathbb{C}, \operatorname{Re}(\beta)>0,|\arg (\gamma)|<\pi$,

$$
\begin{aligned}
& \int_{0}^{\infty} \frac{x^{m} \log ^{k}(a x)}{\left(\beta^{2}+x^{2}\right)(\gamma+x)} \mathrm{d} x \\
& =\frac{\gamma \pi^{k+1} e^{(1 / 2) i \pi(k+m)} \beta^{m-1} \Phi\left(-e^{i m \pi},-k,\left(\left(-2 i \log (a)-t 2 n i q h_{l} \circ \operatorname{og} x(\beta)\right)+\pi / 2 \pi\right)\right)}{\beta^{2}+\gamma^{2}} \\
& -\frac{(i \pi)^{k+1} e^{(i \pi m / 2)} \beta^{m} \Phi\left(e^{i m \pi},-k,\left(\left(-2 i \log (a)-t 2 n i q h_{l} \operatorname{og} x(\beta)\right)+\pi / 2 \pi\right)\right)}{\beta^{2}+\gamma^{2}} \\
& +\frac{(2 i \pi)^{k+1} e^{i \pi m} \gamma^{m} \Phi\left(e^{2 i m \pi},-k,((-i \log (a)-\operatorname{tinq} \log (\gamma))+\pi / 2 \pi)\right)}{\beta^{2}+\gamma^{2}} .
\end{aligned}
$$

Proof. Since the right-hand side of equation (4) is equal to the sum of equations (10), (13), and (18), we may equate the left-hand sides to get the stated result.

Note when $m$ is replaced by $m-1$, we get the Mellin transform representation.

\section{Derivation of an Entry in Gradshteyn and Ryzhik}

In this section, we will derive equation (3.263) in [6]. Using equation (19) and setting $k=0$ and simplifying, we obtain

$$
\int_{0}^{\infty} \frac{x^{m}}{\left(\beta^{2}+x^{2}\right)(\gamma+x)} \mathrm{d} x=\frac{\pi \csc (\pi m)\left(\beta^{m}(\beta \cos (\pi m / 2)+\gamma \sin (\pi m / 2))-\beta \gamma^{m}\right)}{\beta\left(\beta^{2}+\gamma^{2}\right)}
$$

from entry (2) below Table $(64: 12: 7)$ in [9].

\section{Definite Integral in terms of the Hypergeometric Function}

In this section, we will look at derivations in terms of the Hypergeometric function which can be derived from the
Lerch function and apply it to get special cases. We will make use of equation (9.559) in [6] and Table 18-1 in [9] for evaluation of specific values of the parameters.

Using equation (19) and setting $k=1$ and simplifying, we obtain

$$
\begin{aligned}
& \int_{0}^{\infty} \frac{x^{m}}{\left(\beta^{2}+x^{2}\right)(\gamma+x) \log (a x)} \mathrm{d} x \\
& =-\frac{i \gamma e^{(i \pi m / 2)} \beta^{m-1} \Phi\left(-e^{i m \pi}, 1,\left(\left(-2 i \log (a)-t 2 n i q h_{l} \circ \operatorname{og} x(\beta)\right)+\pi / 2 \pi\right)\right)}{\beta^{2}+\gamma^{2}} \\
& \quad-\frac{e^{(i \pi m / 2)} \beta^{m} \Phi\left(e^{i m \pi}, 1,\left(\left(-2 i \log (a)-t 2 n i q h_{l} \circ g x(\beta)\right)+\pi / 2 \pi\right)\right)}{\beta^{2}+\gamma^{2}} \\
& +\frac{e^{i \pi m} \gamma^{m} \Phi\left(e^{2 i m \pi}, 1,((-i \log (a)-t i n q h \log (\gamma))+\pi / 2 \pi)\right)}{\beta^{2}+\gamma^{2}} .
\end{aligned}
$$


7.1. Entry H1. Using (21) and setting $a=-1, \beta=1, \gamma=1$, and $m=1 / 2$ and simplifying, we obtain

$$
\int_{0}^{\infty} \frac{\sqrt{x}}{(x+1)\left(x^{2}+1\right) \log (-x)} \mathrm{d} x=\frac{1}{2} i \log (6+4 \sqrt{2})-i \sqrt{2} .
$$

Next, we rationalize the denominator on the left-hand side and simplify to obtain

$$
\begin{aligned}
& \int_{0}^{\infty} \frac{\pi \sqrt{x}}{(x+1)\left(x^{2}+1\right)\left(\log ^{2}(x)+\pi^{2}\right)} \mathrm{d} x=\sqrt{2}-\frac{1}{2} \log (6+4 \sqrt{2}), \\
& \int_{0}^{\infty} \frac{\sqrt{x} \log (x)}{(x+1)\left(x^{2}+1\right)\left(\log ^{2}(x)+\pi^{2}\right)} \mathrm{d} x=0 .
\end{aligned}
$$

7.2. Entry H2. Using equation (21), setting $\beta=\gamma=1$, formulating a second equation by replacing $m$ by $p$, and taking the difference and simplifying, we obtain

$$
\int_{0}^{\infty} \frac{x^{m}-x^{p}}{(x+1)\left(x^{2}+1\right) \log (x)} \mathrm{d} x=\frac{1}{2} \log \left(\frac{(\tan (\pi m / 4)+1)^{2} \cos ^{2}(\pi p / 4)}{\sin (\pi p / 2)+1}\right)
$$

7.3. Entry H3. Using equation (21), taking the first partial derivative with respect to $m$, and setting $\beta=\gamma=1, a=e^{\pi i}$, and $m=-1 / 2$ simplifying, we obtain

$$
\begin{aligned}
& \int_{0}^{\infty} \frac{\log (x)}{\sqrt{x}(x+1)\left(x^{2}+1\right)\left(\log ^{2}(x)+\pi^{2}\right)} \mathrm{d} x=\frac{\sqrt{2} \pi-\left(\pi^{2} / 2\right)}{\pi}, \\
& \int_{0}^{\infty} \frac{\log ^{2}(x)}{\sqrt{x}(x+1)\left(x^{2}+1\right)\left(\log ^{2}(x)+\pi^{2}\right)} \mathrm{d} x=\frac{\pi}{2}-\frac{1}{2} \pi \log (2),
\end{aligned}
$$

after rationalizing the real and imaginary parts.

7.4. Entry H4. Using equation (21), taking the first partial derivative with respect to $m$, and setting $\beta=\gamma=1, a=e^{\pi i / 2}$, and $m=-1 / 2$ simplifying, we obtain

$$
\begin{array}{r}
\int_{0}^{\infty} \frac{\log ^{2}(x)}{\sqrt{x}(x+1)\left(x^{2}+1\right)\left(4 \log ^{2}(x)+\pi^{2}\right)} \mathrm{d} x=\frac{1}{64} \pi(8-2 \sqrt{2} \pi+\sqrt{2} \log (17+12 \sqrt{2})) \\
\int_{0}^{\infty} \frac{\log (x)}{\sqrt{x}(x+1)\left(x^{2}+1\right)\left(4 \log ^{2}(x)+\pi^{2}\right)} \mathrm{d} x=\frac{\log (4)-\pi}{16 \sqrt{2}},
\end{array}
$$

after rationalizing the real and imaginary parts.

\section{Derivation of New Malmsten-Type Integrals over the Domain $x \in[0, \infty)$}

8.1. Definite Integrals of $\log (\log (x))$ with a Cubic Denominator. In this section, we will use equation (19) to derive a Table of Malmsten's type $\log (\log (x))$ integrals [4] in terms of the $\log$ gamma function $\log (\Gamma(x))$ and the fundamental constant $\pi$. The forms, which we will derive, are given by

$$
\int_{0}^{\infty} \frac{\log (\log (x))}{\left(\beta^{2}+x^{2}\right)(\gamma+x)} d x
$$

and we use equations (1.10.10) in [10], $(64: 13: 3)$ in [9], and (24.14.2) in [11] for simplifying the formula derived.

8.2. Entry M1. Using equation (19) and setting $m=0$ and $a=1$ followed by taking the first partial derivative with respect to $k$ and then setting $k=0$ and simplifying, we obtain 


$$
\begin{aligned}
& \int_{0}^{\infty} \frac{\log (\log (x))}{\left(\beta^{2}+x^{2}\right)(\gamma+x)} \mathrm{d} x=\frac{\pi \gamma}{2 \beta\left(\beta^{2}+\gamma^{2}\right)}(\log (\pi) \\
& \left.+2 \log \left(\frac{(1+i) \Gamma((3 / 4)-(i \log (\beta) / 2 \pi))}{\Gamma((\pi-2 i \log (\beta)) / 4 \pi)}\right)\right) \\
& \quad-2 \beta(\log (2 \pi) \log (\gamma) \\
& \left.\quad+2 i \pi \log \left(\frac{\sqrt[4]{2 \pi} \sqrt[4]{\gamma \Gamma}((\pi-i \log (\gamma)) / 2 \pi)}{\sqrt{-\pi-2 i \log (\beta)} \sqrt{\Gamma(-(i \log (\beta) / \pi)-(1 / 2))}}\right)\right) \\
& \quad+\beta(2 \log (\pi)+i \pi) \log (\beta) .
\end{aligned}
$$

8.3. Entry M2. Next, using equation (28) and setting $\beta=$ $\gamma=1$ and simplifying, we obtain

$$
\int_{0}^{\infty} \frac{\log (\log (x))}{(x+1)\left(x^{2}+1\right)} \mathrm{d} x=\frac{1}{8} i \pi\left(\pi+\log (4)+\log \left((2 \pi)^{-2 i}\left(\frac{3 \Gamma(-(3 / 4))}{\Gamma(-(1 / 4))}\right)^{4 i}\right)\right)
$$

8.4. Entry M3. Next, using equation (28) and setting $\beta=1$ and $\gamma=\sqrt{2}$ and simplifying, we obtain

$$
\begin{gathered}
\int_{0}^{\infty} \frac{\log (\log (x))}{(x+\sqrt{2})\left(x^{2}+1\right)} \mathrm{d} x=\frac{1}{6}(-\log (2) \log (2 \pi) \\
+\pi\left(\sqrt{2} \log (\pi)+2 \sqrt{2} \log \left(\frac{(1+i) \Gamma(3 / 4)}{\Gamma(1 / 4)}\right)\right. \\
\left.\left.-4 i \log \left(-\frac{\Gamma((1 / 2)-(i \log (2) / 4 \pi))}{\sqrt[8]{2} \sqrt{\pi}}\right)\right)\right) .
\end{gathered}
$$

$$
\begin{gathered}
\int_{0}^{\infty} \frac{\log (\log (x))}{(x+\sqrt{3})\left(x^{2}+1\right)} \mathrm{d} x=\frac{1}{8}(-\log (3) \log (2 \pi) \\
+\pi\left(\sqrt{3} \log (\pi)+2 \sqrt{3} \log \left(\frac{(1+i) \Gamma(3 / 4)}{\Gamma(1 / 4)}\right)\right. \\
\left.\left.-4 i \log \left(-\frac{\sqrt[8]{3} \Gamma((1 / 2)-(i \log (3) / 4 \pi))}{\sqrt[4]{2} \sqrt{\pi}}\right)\right)\right) .
\end{gathered}
$$

8.6. Entry M5. Next, using equation (28) and setting $\beta=2^{1 / 4}$ and $\gamma=\sqrt{3}$ and simplifying, we obtain

8.5. Entry M4. Next, using equation (28) and setting $\beta=1$ and $\gamma=\sqrt{3}$ and simplifying, we obtain

$$
\begin{aligned}
& \int_{0}^{\infty} \frac{\log (\log (x))}{(x+\sqrt{3})\left(x^{2}+\sqrt{2}\right)} \mathrm{d} x=\frac{1}{8}(3+\sqrt{2})(\log (4) \log (\pi)-4 \log (3) \log (2 \pi)) \\
& +\frac{\pi}{8(3+\sqrt{2})}\left(i \log (2)+2\left(2^{3 / 4} \sqrt{3}-2 i\right) \log (\pi)\right. \\
& +42^{3 / 4} \sqrt{3} \log \left(\frac{(1+i) \Gamma((3 / 4)-(i \log (2) / 8 \pi))}{\Gamma((1 / 4)-(i \log (2) / 8 \pi))}\right) \\
& \left.-2 i \log \left(\frac{192 \Gamma((1 / 2)-(i \log (3) / 4 \pi))^{8}}{(\log (2)-2 i \pi)^{4} \Gamma(-(1 / 2)-(i \log (2) / 4 \pi))^{4}}\right)\right) .
\end{aligned}
$$

8.7. Entry M6. Using equation (28) and setting $\beta=i$ and simplifying, we obtain 


$$
\begin{array}{r}
\int_{0}^{\infty} \frac{\log (\log (x))}{\left(x^{2}-1\right)(\gamma+x)} \mathrm{d} x=\frac{1}{\gamma^{2}-1}(-\log (2 \pi) \log (\gamma) \\
\left.+2 i \pi \log \left(\frac{\sqrt{\pi} 2^{(1 / 4)-(\gamma / 4)} e^{-(1 / 8) i \pi(\gamma-1)}}{\sqrt[4]{\gamma} \Gamma((\pi-i \log (\gamma)) / 2 \pi)}\right)\right) .
\end{array}
$$

The singularity is at $x=1$ which includes the $\log (\log (x))$ term, so we have to use a principle value integral here.

8.8. Entry $M 7$. Using equation (33) and applying L'Hopital's rule as $\gamma \longrightarrow 1$ and simplifying, we obtain

$$
\int_{0}^{\infty} \frac{\log (\log (x))}{(x-1)(x+1)^{2}} \mathrm{~d} x=\frac{1}{8}\left(4 \gamma+\pi^{2}-2 i \pi(1+\log (2))+\log \left(\frac{16}{\pi^{4}}\right)\right),
$$

where $\gamma$ is Euler's constant.

8.9. Entry M8. Using equation (33) and applying L'Hopital's rule to the right-hand side as $\gamma \longrightarrow-1$ and simplifying, we obtain

$$
\int_{0}^{\infty} \frac{\log (\log (x))}{(x-1)^{2}(x+1)} \mathrm{d} x=\frac{1}{8}\left(4 \gamma-\pi^{2}+\log \left(2^{-4+2 i \pi}\right)-4 \log (\pi)\right) .
$$

8.10. Entry M9. Using equation (33) and setting $\gamma=2$ and simplifying, we obtain

$$
\int_{0}^{\infty} \frac{\log (\log (x))}{(x+2)\left(x^{2}-1\right)} \mathrm{d} x=\frac{1}{3}\left(-\log (2) \log (2 \pi)+2 i \pi \log \left(\frac{e^{-(i \pi / 8)} \sqrt{\pi / 2}}{\Gamma((\pi-i \log (2)) / 2 \pi)}\right)\right) .
$$

8.11. Entry M10. Using equation (33) and setting $\gamma=-2$ and simplifying, we obtain

$$
\int_{0}^{\infty} \frac{\log (\log (x))}{(x-2)\left(x^{2}-1\right)} d x=\frac{1}{12}\left(-4 \log (2) \log (2 \pi)-\pi\left(\pi+8 i \log \left(\Gamma\left(1-\frac{i \log (2)}{2 \pi}\right)\right)\right)\right)
$$

8.12. Entry M11. Using equation (33), then taking the first partial derivative with respect to $\gamma$, and setting $\gamma=1$ and simplifying, we obtain

$$
\int_{0}^{\infty} \frac{\log (\log (x))}{(x-1)(x+1)^{3}} \mathrm{~d} x=\frac{1}{16}\left(\pi^{2}-i \pi(5+\log (4))+8\left(\gamma+\log \left(\frac{2}{\pi}\right)\right)\right) .
$$

8.13. Entry M12. Using equation (33), then taking the first partial derivative with respect to $\gamma$, and setting $\gamma=-1$ and simplifying, we obtain

$$
\int_{0}^{\infty} \frac{\log (\log (x))}{(x-1)^{3}(x+1)} \mathrm{d} x=\frac{1}{48}\left(-24 \gamma+3 \pi(\pi+3 i)+\log \left(64^{4-i \pi}\right)+24 \log (\pi)\right)
$$

8.14. Entry M13. Using equation (33), then taking the second partial derivative with respect to $\gamma$, and setting $\gamma=1$ and simplifying, we obtain 


$$
\int_{0}^{\infty} \frac{\log (\log (x))}{(x-1)(x+1)^{4}} \mathrm{~d} x=\frac{-28 \zeta(3)+3 \pi^{4}-i \pi^{3}(29+\log (64))+40 \pi^{2}(\gamma+\log (2 / \pi))}{96 \pi^{2}}
$$

8.15. Entry M14. Using equation (33), then taking the second partial derivative with respect to $\gamma$, and setting $\gamma=-1$ and simplifying, we obtain

$$
\int_{0}^{\infty} \frac{\log (\log (x))}{(x-1)^{4}(x+1)} d x=\frac{1}{96}\left(-\frac{4 \zeta(3)}{\pi^{2}}+40 \gamma-3 \pi^{2}+i \pi(\log (64)-23)-40 \log (2 \pi)\right)
$$

8.16. Definite Integrals of $\log (\log (x))$ and a Rational Quotient Function. In this section, we again look at Malmsten integrals [4] of the form

$$
\int_{0}^{\infty} \frac{\log (\log (x))\left(x^{n}-x^{m}\right)}{\left(\beta^{2}+x^{2}\right)(\gamma+x)} \mathrm{d} x \text {. }
$$

Using equation (19) and formulating a second equation by replacing $m$ by $n$, then taking the difference followed by taking the first partial derivative with respect to $k$, and then setting $k=0$ and simplifying in terms of the derivative of the Lerch function, we obtain

l

$$
\begin{aligned}
& \int_{0}^{\infty} \frac{\log (\log (x))\left(x^{n}-x^{m}\right)}{\left(\beta^{2}+x^{2}\right)(\gamma+x)} \mathrm{d} x=\frac{\pi}{4 \beta\left(\beta^{2}+\gamma^{2}\right)}\left(-i(\pi-2 i \log (\pi)) \beta^{m+1} \csc \left(\frac{\pi m}{2}\right)\right. \\
& +\beta(2 i \pi+\log (16)+4 \log (\pi)) \gamma^{m} \csc (\pi m) \\
& +(2 \log (\pi)+i \pi)\left(\beta^{n}\left(\beta \csc \left(\frac{\pi n}{2}\right)+\gamma \sec \left(\frac{\pi n}{2}\right)\right)\right. \\
& \left.-\gamma \beta^{m} \sec \left(\frac{\pi m}{2}\right)\right) \\
& \left.-2 i \beta\left(\pi-i \log \left(4 \pi^{2}\right)\right) \gamma^{n} \csc (\pi n)\right) \\
& +\frac{\pi \gamma e^{(i \pi m / 2)} \beta^{m-1} \Phi \prime\left(-e^{i \pi m}, 0,((1 / 2)-(i \log (\beta) / \pi))\right)}{\beta^{2}+\gamma^{2}} \\
& -\frac{i \pi e^{(i \pi m / 2)} \beta^{m} \Phi \prime\left(e^{i \pi m}, 0,((1 / 2)-(i \log (\beta) / \pi))\right)}{\beta^{2}+\gamma^{2}} \\
& +\frac{2 i \pi e^{i \pi m} \gamma^{m} \Phi \prime\left(e^{2 i \pi m}, 0,((\pi-i \log (\gamma)) / 2 \pi)\right)}{\beta^{2}+\gamma^{2}} \\
& -\frac{\pi \gamma e^{(i \pi n / 2)} \beta^{n-1} \Phi \prime\left(-e^{i \pi n}, 0,((1 / 2)-(i \log (\beta) / \pi))\right)}{\beta^{2}+\gamma^{2}} \\
& +\frac{i \pi e^{(i \pi n / 2)} \beta^{n} \Phi \prime\left(e^{i \pi n}, 0,((1 / 2)-(i \log (\beta) / \pi))\right)}{\beta^{2}+\gamma^{2}} \\
& -\frac{2 i \pi e^{i \pi n} \gamma^{n} \Phi \prime\left(e^{2 i \pi n}, 0,((\pi-i \log (\gamma)) / 2 \pi)\right)}{\beta^{2}+\gamma^{2}} .
\end{aligned}
$$


8.17. Enrty MQ1. Using equation (43), we set $\beta=\gamma=1$, $m=1 / 2$, and $n=1 / 3$ simplifying to obtain

$$
\begin{aligned}
\int_{0}^{\infty} \frac{(\sqrt[3]{x}-\sqrt{x}) \log (\log (x))}{(x+1)\left(x^{2}+1\right)} \mathrm{d} x= & -\pi \Phi^{\prime}\left(-1,0, \frac{1}{2}\right)+\frac{1}{2} \sqrt{[4]}-1 \pi \Phi^{\prime}\left(-i, 0, \frac{1}{2}\right) \\
& -\frac{1}{2}(-1)^{3 / 4} \pi \Phi^{\prime}\left(i, 0, \frac{1}{2}\right) \\
& -\frac{1}{2} \sqrt[6]{-1} \pi \Phi^{\prime}\left(-\sqrt[3]{-1}, 0, \frac{1}{2}\right) \\
& +\frac{1}{2}(-1)^{2 / 3} \pi \Phi^{\prime}\left(\sqrt[3]{-1}, 0, \frac{1}{2}\right) \\
& -(-1)^{5 / 6} \pi \Phi^{\prime}\left((-1)^{2 / 3}, 0, \frac{1}{2}\right) \\
& +\frac{i \pi^{2}}{2}-\frac{i \pi^{2}}{2 \sqrt{2}}-\frac{i \pi^{2}}{4 \sqrt{3}}+\frac{1}{12} \pi \log (64) \\
& +\pi \log (\pi)-\frac{\pi \log (\pi)}{\sqrt{2}}-\frac{\pi \log (4 \pi)}{2 \sqrt{3}} .
\end{aligned}
$$

\section{Definite Integrals of Bierens De Haan Type}

In this section, we will derive definite integrals similar to works published in [12] given by

$$
\begin{aligned}
& \int_{0}^{\infty} \frac{\log (\log (x))}{(x+\gamma)\left(x^{2}+\beta^{2}\right) \sqrt{\log (x)}} \mathrm{d} x, \\
& \int_{0}^{\infty} \frac{\sqrt{\log (x)} \log (\log (x))}{(x+\gamma)\left(x^{2}+\beta^{2}\right)} \mathrm{d} x,
\end{aligned}
$$

and use equation $(64: 13: 3)$ in [9] and (25.14.2) in [11] for simplifying the derived formula.

9.1. Entry B1. Using equation (19) and setting $a=1$ and $m=$ 0 and simplifying, we obtain

$$
\begin{aligned}
& \int_{0}^{\infty} \frac{\log ^{k}(x)}{\left(\beta^{2}+x^{2}\right)(\gamma+x)} \mathrm{d} x=\frac{e^{(i \pi k / 2)} \pi^{k+1}}{\beta\left(\beta^{2}+\gamma^{2}\right)}\left(2 ^ { k } \left(\gamma \zeta\left(-k, \frac{\pi-2 i \log (\beta)}{4 \pi}\right)\right.\right. \\
& \left.-\gamma \zeta\left(-k, \frac{3}{4}-\frac{i \log (\beta)}{2 \pi}\right)+2 i \beta \zeta\left(-k,\left(\frac{\pi-i \log (\gamma)}{2 \pi}\right)\right)\right) \\
& \left.-i \beta \zeta\left(-k, \frac{1}{2}-\frac{i \log (\beta)}{\pi}\right)\right) .
\end{aligned}
$$

9.2. Entry B2. Next, we take the first partial derivative with respect to $k$ and set $k=-1 / 2$ and $\beta=\gamma=1$ and simplify to obtain 


$$
\begin{aligned}
\int_{0}^{\infty} \frac{\log (\log (x))}{(x+1)\left(x^{2}+1\right) \sqrt{\log (x)}} \mathrm{d} x= & \frac{1}{8} \sqrt[4]{-1} \sqrt{\pi}\left(2 i \sqrt{2} \zeta^{\prime}\left(\frac{1}{2}, \frac{1}{4}\right)-2 i \sqrt{2} \zeta\left(\frac{1}{2}, \frac{3}{4}\right)\right. \\
& +\sqrt{2} \zeta\left(\frac{1}{2}, \frac{1}{4}\right)(\pi-2 i \log (2 \pi)) \\
& -\sqrt{2} \zeta\left(\frac{1}{2}, \frac{3}{4}\right)(\pi-2 i \log (2 \pi))+(2 \sqrt{2}-3) \\
& \left.\zeta\left(\frac{1}{2}\right)\left(2 \gamma+(1-2 i) \pi+\log \left(\frac{64}{\pi^{2}}\right)\right)\right)
\end{aligned}
$$

where $\gamma$ is Euler's constant given by equation (9.73) in [6].

9.3. Entry B3. Another example is by once again using equation (19) taking the first partial derivative with respect to $k$ setting $k=1 / 2$ and $\beta=\gamma=1$ and simplifying to obtain

$$
\begin{aligned}
\int_{0}^{\infty} \frac{\sqrt{\log (x)} \log (\log (x))}{(x+1)\left(x^{2}+1\right)} \mathrm{d} x= & \frac{1}{32} \sqrt[4]{-1 \pi^{3 / 2}}\left(-16 \sqrt{2} \zeta^{\prime}\left(-\frac{1}{2}, \frac{1}{4}\right)+16 \sqrt{2} \zeta^{\prime}\left(-\frac{1}{2}, \frac{3}{4}\right)\right. \\
& -48 i \zeta^{\prime}\left(-\frac{1}{2}\right)+40 i \sqrt{2} \zeta^{\prime}\left(-\frac{1}{2}\right) \\
& +8 \sqrt{2} \zeta\left(-\frac{1}{2}, \frac{3}{4}\right) \log \left(\frac{1}{4 \pi^{2}}\right) \\
& \left.+8 \sqrt{2} \zeta\left(-\frac{1}{2}, \frac{1}{4}\right) \log \left(4 \pi^{2}\right)+6 \zeta\left(\frac{3}{2}\right)-5 \sqrt{2} \zeta\left(\frac{3}{2}\right)\right) \\
& -\frac{(-1)^{3 / 4} \pi^{5 / 2}(\zeta(-(1 / 2),(3 / 4))-\zeta(-(1 / 2),(1 / 4)))}{2 \sqrt{2}} \\
& +\frac{1}{16}(-1)^{3 / 4} \sqrt{\pi} \zeta\left(\frac{3}{2}\right)(\sqrt{2} \log (8) \\
& -6 \log (\pi)+5 \sqrt{2} \log (\pi)) .
\end{aligned}
$$

\section{Table of Mellin Transforms}

In this section, we will use equation (19) to derive a table of Mellin transforms not published in current literature.

10.1. Entry MT1. We take the first partial derivative with respect to $\gamma$ and set $\beta=\gamma=a=k=1$ and replace $m$ by $s-1$ and simplify to obtain

$$
\begin{aligned}
\int_{0}^{\infty} \frac{x^{s-1} \log (x)}{(x+1)^{2}\left(x^{2}+1\right)} \mathrm{d} x= & -\frac{1}{2} \pi \csc (\pi s)(-\pi(s-2) \cot (\pi s) \\
& \left.+\pi \sin ^{3}\left(\frac{\pi s}{2}\right) \csc (\pi s)+1\right)
\end{aligned}
$$

from entry (1) below Table $(64: 12: 7)$ in [9].
10.2. Entry MT2. We take the first partial derivative with respect to $\beta$ and set $\beta=\gamma=a=k=1$ and replace $m$ by $s-1$ and simplify to obtain

$$
\begin{aligned}
& \int_{0}^{\infty} \frac{x^{s-1} \log (x)}{(x+1)\left(x^{2}+1\right)^{2}} \mathrm{~d} x=\frac{1}{16} \pi \csc ^{2}(\pi s)\left((3 \pi(s-2)-2) \sin \left(\frac{\pi s}{2}\right)\right. \\
& -(\pi(s-2)+2) \sin \left(\frac{3 \pi s}{2}\right)+(3 \pi(s-3)+2) \cos \left(\frac{\pi s}{2}\right) \\
& \left.\quad-4 \pi \cos (\pi s)+(\pi(s-3)-2) \cos \left(\frac{3 \pi s}{2}\right)\right),
\end{aligned}
$$

from entry (1) below Table $(64: 12: 7)$ in [9].

10.3. Entry MT3. We take the first partial derivative with respect to $\gamma$ and set $\beta=\gamma=a=1, k=1 / 2$ and replace $m$ by $s-1$ and simplify to obtain 


$$
\begin{aligned}
\int_{0}^{\infty} \frac{x^{s-1} \sqrt{\log (x)}}{(x+1)^{2}\left(x^{2}+1\right)} \mathrm{d} x= & \left(\frac{1}{4}+\frac{i}{4}\right) \sqrt{\frac{\pi}{2}} e^{(i \pi s / 2)} \sqrt{2} e^{(i \pi s / 2)} 4 i \pi(s-2) \Phi\left(e^{2 i \pi s},-\frac{1}{2}, \frac{1}{2}\right) \\
& \left.\left.+\Phi\left(e^{2 i \pi s}, \frac{1}{2}, \frac{1}{2}\right)\right)-2 \pi \Phi\left(-e^{i \pi s},-\frac{1}{2}, \frac{1}{2}\right)\right) .
\end{aligned}
$$

10.4. Entry MT4. We take the first partial derivative with respect to $a$ and set $\beta=\gamma=a=1$ and $k=1 / 3$ and replace $m$ by $s-1$ and simplify to obtain

$$
\begin{aligned}
& \int_{0}^{\infty} \frac{x^{s-1}}{(x+1)\left(x^{2}+1\right) \log ^{(2 / 3)}(x)} \mathrm{d} x=\frac{1}{2}(-1)^{2 / 3} \sqrt[3]{\pi} e^{(i \pi s / 2)}\left(\Phi\left(-e^{i \pi s}, \frac{2}{3}, \frac{1}{2}\right)\right. \\
& \left.+i \Phi\left(e^{i \pi s}, \frac{2}{3}, \frac{1}{2}\right)\right)-\frac{\sqrt[6]{-1} \sqrt[3]{\pi} e^{i \pi s} \Phi\left(e^{2 i \pi s},(2 / 3),(1 / 2)\right)}{2^{2 / 3}}
\end{aligned}
$$

10.5. Entry MT5. We take the first partial derivatives with respect to $\beta$ then $\gamma$ and set $\beta=\gamma=a=1$ and $k=1 / 2$ and replace $m$ by $s-1$ and simplify to obtain

$$
\begin{aligned}
\int_{0}^{\infty} \frac{x^{s-1} \sqrt{\log (x)}}{(x+1)^{2}\left(x^{2}+1\right)^{2}} \mathrm{~d} x= & \left(\frac{1}{8}+\frac{i}{8}\right) \sqrt{\frac{\pi}{2}} e^{(i \pi s / 2)}\left(2 \pi s \Phi\left(-e^{i \pi s},-\frac{1}{2}, \frac{1}{2}\right)\right. \\
& -6 \pi \Phi\left(-e^{i \pi s},-\frac{1}{2}, \frac{1}{2}\right)-i \Phi\left(-e^{i \pi s}, \frac{1}{2}, \frac{1}{2}\right) \\
& \left.-2 i \pi \Phi\left(e^{i \pi s},-\frac{1}{2}, \frac{1}{2}\right)\right) \\
& +\left(\frac{1}{8}+\frac{i}{8}\right) \sqrt{\pi} e^{i \pi s}\left(4 i \pi s \Phi\left(e^{2 i \pi s},-\frac{1}{2}, \frac{1}{2}\right)\right. \\
& \left.-12 i \pi \Phi\left(e^{2 i \pi s},-\frac{1}{2}, \frac{1}{2}\right)+\Phi\left(e^{2 i \pi s}, \frac{1}{2}, \frac{1}{2}\right)\right) .
\end{aligned}
$$

10.6. Entry MT6. Here, we derive the Mellin transform in terms of the Polylogarithmic function. We set $\beta=i / a$ and $a=1$ and replace $m$ by $s-1$ and simplify to obtain

$$
\begin{aligned}
\int_{0}^{\infty} \frac{x^{s-1} \log ^{k}(x)}{\left(x^{2}-1\right)(\gamma+x)} \mathrm{d} x= & -\frac{e^{(1 / 2) i \pi(k+1)} \pi^{k+1}}{\gamma\left(\gamma^{2}-1\right)}\left(2^{k+1} e^{i \pi s} \gamma^{s} \Phi\left(e^{2 i \pi s},-k, \frac{\pi-i \log (\gamma)}{2 \pi}\right)\right. \\
& \left.+\gamma\left(L i_{-k}\left(-e^{i \pi s}\right)-\gamma L i_{-k}\left(e^{i \pi s}\right)\right)\right),
\end{aligned}
$$

from equation (1.11.14) in [10]. 


\section{Conclusion}

In this article, we derived a table of definite integrals which can be included in current books such as $[1,2,6]$. We found that using our simultaneous contour integral method, we were able to generate very interesting new integral formulae. We also looked at analytic continuity and principle value methods to evaluate integrals when singularities were present. One of the consequences of this work is the production of a table of definite integrals which researchers may use to advance their work if they find any of these formulae applicable. We will be looking at other integrals using this contour integral method for future work. The results presented were numerically verified for both real and imaginary values of the parameters in the integrals using Mathematica by Wolfram.

\section{Data Availability}

No data were used to support this study.

\section{Conflicts of Interest}

The authors declare that they have no conflicts of interest.

\section{Acknowledgments}

This research was supported by NSERC, Canada, under Grant 504070.

\section{References}

[1] Y. A. Brychkov, O. I. Marichev, and N. V. Savischenko, Handbook of Mellin Transforms, CRC Press, Taylor \& Francis Group, Boca Raton, FL, USA, 2019.

[2] A. Erdéyli, W. Magnus, F. Oberhettinger, and F. G. Tricomi, Tables of Integral Transforms, Vol. II, McGraw-Hill Book Company, Inc., New York, NY, USA, 1954.

[3] R. Reynolds and A. Stauffer, "Note on an integral by anatolii prudnikov," AIMS Mathematics, vol. 6, no. 3, pp. 2680-2689, 2021.

[4] C. J. Malmsten, "De integralibus quibusdam definitis seriebusque infinitis (Eng. trans.: on some definite integrals and series)," Journal für die reine und angewandte Mathematik, vol. 38, pp. 1-39, 1849.

[5] R. Reynolds and A. Stauffer, "A method for evaluating definite integrals in terms of special functions with examples," International Mathematical Forum, vol. 15, no. 5, pp. 235-244, 2020.

[6] I. S. Gradshteyn and I. M. Ryzhik, Tables of Integrals, Series and Products, Academic Press, Cambridge, MA, USA, 6 edition, 2000.

[7] H. M. Srivastava and J. Choi, Zeta and Q-Zeta Functions and Associated Series and Integrals, Elsevier Science Publishers, Amsterdam, Netherlands, 2012.

[8] M. Abramowitz and I. A. Stegun, Handbook of Mathematical Functions with Formulas, Graphs, and Mathematical Tables, Dover, New York, NY, USA, 9th edition, 1982.

[9] K. B. Oldham, J. C. Myland, and J. Spanier, An Atlas of Functions: With Equator, the Atlas Function Calculator, Springer, New York, NY, USA, 2nd edition, 2009.
[10] A. Erdéyli, W. Magnus, F. Oberhettinger, and F. G. Tricomi, Higher Transcendental Functions, Vol. I, McGraw-Hill Book Company, Inc., New York, NY, USA, 1953a.

[11] F. W. J. Olver and A. B. Olde Daalhuis, NIST Digital Library of Mathematical Functions, D. W. Lozier, B. I. Schneider, R. F. Boisvert et al., Eds., National Bureau of Standards, Gaithersburg, MD, USA, 2020, http://dlmf.nist.gov/.

[12] D. Bierens De Haan, Nouvelles Tables D’intégrales Définies, Johns Hopkins University Press, Baltimore, MD, USA, 1867. 